\title{
A Note on Specification Property of Dynamical Systems
}

\author{
Nan Li ${ }^{1}$, Lidong Wang ${ }^{1,2,3, ~ *}$, Fengchun Lei ${ }^{1}$ \\ ${ }^{1}$ School of Mathematical Science, Dalian University of Technology, Dalian, China \\ ${ }^{2}$ School of Science, Dalian Nationalities University, Dalian, China \\ ${ }^{3}$ The College of Public Foundation and Innovation and Entrepreneurship, Zhuhai College of Jilin University, Zhuhai, China
}

Email address:

liyufeng177@mail.dlut.edu.cn (Nan Li),wld0707@126.com (Lidong Wang), fclei@dlut.edu.cn (Fengchun Lei)

${ }^{*}$ Corresponding author

\section{To cite this article:}

Nan Li, Lidong Wang, Fengchun Lei. A Note on Specification Property of Dynamical Systems. Mathematics Letters.

Vol. 4, No. 2, 2018, pp. 34-38. doi: 10.11648/j.ml.20180402.12

Received: April 20, 2018; Accepted: May 22, 2018; Published: June 28, 2018

\begin{abstract}
The paper is discussed the sensitive and transitive property of a dynamical system with strong specification property. It is proved that if a dynamical system is sensitive, then it is syndetically sensitive with the same constant of sensitivity. Further, it is given another condition such that if a dynamical system is sensitive, then it is syndetically sensitive with the same constant of sensitivity. Meanwhile, it is stated that if a dynamical system has shadowing property, then it is totally syndetically transitive.
\end{abstract}

Keywords: Sensitive, Specification Property, Syndetically Transitive

\section{Introduction}

Specification property was first introduced by Bowen to give the distribution of periodic points for Axiom A diffeomorphisms [1]. In recent years, many scholars focus on specification property and its related properties. Lampart [2] described some relations between specification property and $\omega$-chaos. Kulczycki [3] studied relations between almost specification property, asymptotic average shadowing property and average shadowing property for compact dynamical systems. Wang at. el. [4] shown that an expansive system with specification property displays a stronger form of $\omega$-chaos. It is proved that there exists a system having almost specification property and zero entropy [5]. Kwietniak [6] examined relations between specification like properties and such notions as: mixing, entropy, the structure of the simplex of invariant measures, and various types of the shadowing property. Kwietniak at. el. [7] stated that the weak specification property neither implies intrinsic ergodicity, nor follows from almost specification. It was proved that a self homeomorphism on property [8].

The paper is organized in the following manner: In Section 2 , it is given preliminaries required for the development of the paper. In Section 3, it is proved that if a dynamical system $(X, T)$ is sensitive with strong specification property, then it is syndetically sensitive with the same constant of sensitivity.
Further, it is given another condition such that if $(X, T)$ is sensitive, then it is syndetically sensitive with the same constant of sensitivity. At least, it is shown that if a dynamical system has $\bar{d}$-shadowing property or $\bar{d}$-shadowing property with strong specification property, then it is totally syndetically transitive.

\section{Basic Definitions}

Let $T: X \rightarrow X$ be a continuous map acting on a compact metric space $(X, d)$.

A subset $S$ of $\mathbb{Z}^{+}$is syndetic if it has a bounded gap, i.e., if there is $N \in \mathbb{N}$ such that $\{i, i+1, \cdots, i+N\} \cap S \neq \varnothing$ for every $i \in \mathbb{Z}^{+} ; S$ is thick if it contains arbitrarily long runs of positive integers, i.e., for every $n \in N$ there exists some $a_{n} \in \mathbb{Z}$ such that $\left\{a_{n}, a_{n}+1, \cdots, a_{n}+n\right\} \subset S$.

Let $(X, T)$ be a dynamical system and let $d$ be an admissible metric on $X$.

According to the classical definition, $T$ is sensitive if there exists $\delta>0$ with the property that for any nonempty open set $U \subset X$, one can find $y, z \subset U$ and $n \in N$ such that $d\left(T^{n}(y), T^{n}(z)\right)>\delta$. It is written this in a slightly different way.

For $U \subset X$ and $\delta>0$, let 


$$
\begin{gathered}
N_{T}(U, \delta)=\{n \in \mathbb{N} \text { : there exist } y, z \in U \\
\text { with } \left.d\left(T^{n}(y), T^{n}(z)\right)>\delta\right\} .
\end{gathered}
$$

It is called that $T$ is syndetically sensitive if there exists $\delta>0$ with the property that for every nonempty open set $U \subset X$, so $N_{T}(U, \delta)$ is syndetic.

Let $X$ be a set and

$$
\Delta_{X}=\{(x, x) \mid x \in X\} .
$$

Then $\Delta X$ is called the diagonal of $X \times X$. A subset $M$ of $X \times X$ is said to be symmetric if $M=M^{T}$, where

$$
M^{T}=\{(y, x) \mid(x, y) \in M\} .
$$

Definition 2.1. A map $T$ is said to have strong specification property if for every symmetric neighborhood $U$ of the diagonal $\Delta X$ there is a positive integer $M$ such that for any finite sequence of points $x_{1}, x_{2}, \cdots, x_{k}$ in $X$, any integers

$$
a_{1} \leq b_{1}<a_{2} \leq b_{2}<\cdots<a_{k} \leq b_{k}
$$

with $a_{j}-b_{j-1} \geq M(2 \leq j \leq k)$ and any $p>M+\left(b_{k}-a_{1}\right)$,

there exists $x \in X$ such that $T^{p}(x)=x$ and

$$
F^{i}\left(x, x_{j}\right) \in U, a_{j} \leq i \leq b_{j}, 1 \leq j \leq k .
$$

Let $\varepsilon_{0}>0$. A function

$$
g: \mathbb{N} \times\left(0, \varepsilon_{0}\right) \rightarrow \mathbb{N}
$$

is called a mistake function if for all $\varepsilon \in\left(0, \varepsilon_{0}\right)$ and all

$$
B_{n}(g ; n, \mathcal{\varepsilon}):=\left\{y \in X: y \in B_{\Lambda}(n, \mathcal{\varepsilon}) \text { for some } \Lambda \in I(g ; n, \varepsilon)\right\}=\bigcup_{\Lambda \in I(g ; n, \varepsilon)} B_{\Lambda}(x, \varepsilon) .
$$

Definition 2.2. The dynamical system $(X, T)$ has almost specification property with mistake function $g$, if for any $\varepsilon_{1}, \cdots, \varepsilon_{m}>0$, there exist integers $k_{g}\left(\varepsilon_{1}\right), \cdots, k_{g}\left(\varepsilon_{m}\right)$

such that for any points $x_{1}, \cdots, x_{m} \in X$, and integers

$$
n_{1} \geq k_{g}\left(\varepsilon_{1}\right), \cdots, n_{m} \geq k_{g}\left(\varepsilon_{m}\right), \text { there exists a point } z \in X
$$

such that

$$
T^{l_{j}}(z) \in B_{n_{j}}\left(g ; x_{j}, \varepsilon_{j}\right), j=1, \cdots, m
$$

where $n_{0}=0$ and $l_{j}=\sum_{s}^{j-1} n_{s}$.

Remark 1. Pfister and Sullivan [9] showed that the strong specification property implies the almost specification property.

For any $A \subset \mathbb{N}$, the upper density of $A$ is defined by

$$
\bar{d}(A):=\limsup _{n \rightarrow \infty} \frac{1}{n}|A \bigcap\{0,1, \cdots, n-1\}| .
$$

Replacing limsup with liminf in (1) gives the definition of

$$
n \in \mathbb{N}, g(n, \mathcal{\varepsilon}) \leq g(n+1, \mathcal{\varepsilon})
$$

and

$$
\lim _{n} \frac{g(n, \mathcal{\varepsilon})}{n}=0
$$

Given a mistake function $g$, if $\varepsilon \geq \varepsilon_{0}$, it is defined $g(n, \varepsilon)=g\left(n, \varepsilon_{0}\right)$.

Let $g$ be a mistake function and $\varepsilon>0$. For $n \in \mathbb{N}$ large enough such that

$$
g(n, \varepsilon)<\varepsilon
$$

it is defined

$$
I(g ; n, \mathcal{E}):=\{\Lambda \subset\{0,1, \cdots, n-1\}: \# \Lambda \geq n-g(n, \mathcal{E})\},
$$

where $\# \Lambda$ denotes the cardinality of $\Lambda$.

For a finite set of indices

$$
\Lambda \subset\{0,1, \cdots, n-1\},
$$

it is defined the Bowen distance of $x, y \in X$ along $\Lambda$ by

$$
d_{\Lambda}(x, y):=\max _{j \in \Lambda}\left\{d\left(T^{j} x, T^{j} y\right)\right\}
$$

and the Bowen ball of radius $\mathcal{E}$ centered at $x$ by

$$
B_{\Lambda}(x, \mathcal{E}):=\left\{y \in X: d_{\Lambda}(x, y)<\mathcal{E}\right\} .
$$

When $g(n, \mathcal{E})<n$, it is defined the $(g ; n, \mathcal{E})$ Bowen ball centered at $x$ as

$\underline{d}(A)$, the lower density of $A$. If there exists a number $d(A)$ such that $\bar{d}(A)=\underline{d}(A)=d(A)$ then it is said that the set $A$ has density $d(A)$. Fix any $\alpha \in[0,1)$ and denote by $\mathrm{M}_{\alpha}$ (resp. $\mathbf{M}^{\alpha}$ ) the family consisting of sets $A \subset \mathbb{N}$ with $\underline{d}(A)>\alpha$ (resp. $\bar{d}(A)>\alpha)$. It is denoted by $\mathrm{M}_{\alpha}$ the family of sets with $\underline{d}(A)>\alpha$. Clearly $\hat{\mathrm{M}}_{1}$ consists of sets $A$ with $d(A)=1$

Definition 2.3. A dynamical system $(X, T)$ has (ergodic) $F-$ shadowing property if, for any $\varepsilon>0$ there is $\delta>0$ such that every $\delta$-ergodic pseudo-orbit $\xi$ is $F-\mathcal{E}$-shadowed by some point $z \in X$, i.e.

$$
\Lambda(z, \xi, \varepsilon) \in F .
$$

In the special case of $F=\hat{\mathbf{M}}_{1}$ (resp., $F=\mathrm{M}_{0}$ and $M^{\frac{1}{2}}$ ), it is said that $(X, T)$ has the ergodic shadowing property (resp., 
$\bar{d}$-shadowing property and $\underline{d}$-shadowing property).

\section{Specification Property of Dynamical Systems}

A dynamical system $(X, T)$ is a minimal system (or $T$ is a minimal map) if the $T$-orbit of every $x \in X$ is dense in $X$. It is easy to see that $(X, T)$ is a minimal system if $(X, T)$ has no proper, nonempty, closed $T$-invariant subset. Here, for a subset $Y, T$-invariant means $T(Y) \subset Y$. In a dynamical system $(X, T)$, an element $x \in X$ is called a minimal point if the dynamical system $\left(\overline{O_{T}(x)},\left.T\right|_{O_{T}(x)}\right)$ is minimal. Note that every periodic point is a minimal point, and that every minimal point is a recurrent point.

Theorem 3.1. Let $T$ has strong specification property, if $T$ is sensitive, then $T$ is syndetically sensitive with the same constant of sensitivity.

Lemma 3.2 ([8]). If $T: X \rightarrow X$ has strong specification property, then $T$ is Devaney chaotic.

Proof of Theorem 3.1. By Lemma 3.2 it is easy to see that $T$ is dense of periodic points. Suppose that $T$ is sensitive with constant $\delta>0$. Let $U \subset X$ be a nonempty open set. It is well known that $k \in N_{T}(U, \delta)$ for som $k \in \mathbb{N}$. Since $(X, T)$ has a dense set of minimal points, the product system $(X \times X, T \times T)$ also has a dense set of minimal points [10]. Therefore it can be chosen $y, z \in U$ such that $(y, z)$ is a minimal point for $T \times T$ and such that

$$
d\left(T^{k}(y), T^{k}(z)\right)>\delta .
$$

Let $V, W \subset X$ be small enough open neighborhoods of $T^{k}(y)$ and $T^{k}(z)$, respectively, with $d(v, w)>\delta$ for every $v \in V$ and $w \in W$. In the system $(X \times X, T \times T)$, the open set $V \times W$ is a neighborhood of the minimal point $\left(T^{k}(y), T^{k}(z)\right)$.

Therefore, the set $N_{T \times T}\left(\left(T^{k}(y), T^{k}(z)\right), V \times W\right)$ is syndetic. By the choice of $V$ and $W$, so

$$
N_{T \times T}\left(\left(T^{k}(y), T^{k}(z)\right), V \times W\right)+k \subset N_{T}(U, \delta) .
$$

Therefore, $N_{T}(U, \delta)$ is also syndetic.

Theorem 3.3. Let $T$ has almost specification property and has an invariant measure with full support. If $T$ is sensitive, then $T$ is syndetically sensitive with the same constant of sensitivity. The following lemma follows from the ergodic theorem.

Lemma 3.4 ([11]). If $(X, T)$ is a compact dynamical system, then for every nonempty open set $U \subset X$ there exists a point $x \in X$ such that $d(N(x, U))=\xi(U)$.

Lemma 3.5 ([3]). Let $(X, T)$ be a compact dynamical system. An open set $U \subset X$ is universally null if and only if $\xi(U)=0$, or equivalently $d(N(x, U))=0$ for every $x \in X$.

Proof of Theorem 3.3. Fix any nonempty open set $U$. there is $\varepsilon>0$ and nonempty open sets $W \subset V \subset U$ such that the $\varepsilon$ -neighborhood of $\bar{W}$ is contained in $V$ and $\bar{V} \subset U$. Since $U$ is not universally null, it is concluded from Lemma 3.4 and Lemma 3.5 that there is $\gamma>0$ and a point $x \in W$ such that $d(N(x, W))=\gamma$. So

$$
\# d(N(x, W) \mid n) \geq \frac{n \gamma}{2} \text { for every } n \in N .
$$

Using the almost specification property, there is an $M>0$ such that for all $m>M$, there exists $g(\varepsilon, m) \geq \frac{m \gamma}{2}$.

Let $n=\max \left\{N, M, k_{g}(\varepsilon)\right\}$. Let $\left\{x_{j}\right\}_{j=0}^{\infty},\left\{\varepsilon_{j}\right\}_{j=0}^{\infty},\left\{n_{j}\right\}_{j=0}^{\infty}$

be constant sequences, where $x_{j}=x, \varepsilon_{j}=\varepsilon, n_{j}=n$ for every $j \in \mathbb{N}$. By ([3], Lemma 3.4$)$ there is a point $y_{\text {such }}$ that $T^{j n}(y) \in B_{n}(g ; n, \mathcal{\varepsilon})$ for every $j \in \mathbb{N}$. It is claimed that $N(y, V)$ is syndetic (has gaps bounded by $2 n$ ). Assume conversely that $T^{l}(y) \notin V$ for $2 n$ consecutive indices $l$. In particular, for some $j \geq 0$ and every $0 \leq i<n$ such that $T^{i}(x) \in W$ it necessarily has $\varsigma\left(T^{j n+i}(z), T^{i}(x)\right) \geq \varepsilon$. But this leads to a contradiction: $\frac{n \gamma}{2} \leq g(\varepsilon, n)<\frac{n \gamma}{2}$. Therefore $N(y, V)$ is syndetic with gaps bounded by $2 n$ as claimed. By the Auslander-Ellis Theorem ([12], Theorem 8.7) there is a minimal point $2 n$ proximal to $y$. It is easy to see that $T^{l}(z) \in U$ for some $l \geq 0$. Since $T^{l}(z)$ is also a minimal point the proof is finished.

Applying the proof process of Theorem 3.1, the Theorem 3.3 is proved.

Theorem 3.6. Let $(X, T)$ be a dynamical system with strong specification property. If $(X, T)$ has the $\bar{d}$-shadowing property or $d_{-}$-shadowing property, then $(X, T)$ is totally syndetically transitive.

Lemma 3.7. If $(X, T)$ is a topologically transitive system with a dense set of minimal points, then $(X, T)$ is totally syndetically transitive.

Proof. Let $U, V$ be non-empty open subsets of $X$ containing points $y_{1}$ and $y_{2}$, respectively. Choose a symmetric neighborhood $U$ of the diagonal $\Delta_{X}$ such that

$$
U\left[y_{1}\right]=\left\{y \in X \mid\left(y_{1}, y\right) \in U\right\} \subset V
$$

and

$$
U\left[y_{2}\right]=\left\{y \in X \mid\left(y_{2}, y\right) \in U\right\} \subset W .
$$

Choose a positive integer $M(U)$ as in the definition of TSP. For any sequence,

$$
a_{1}=b_{1}=0<a_{2} \leq b_{2}<\cdots<a_{k} \leq b_{k}
$$

with $a_{j}-b_{j-1} \geq M, j=2,3, \cdots, k$ and

$x_{1}, x_{2}, \cdots x_{k} \in X$, there exists a $z \in X$ such that $T^{p}(z)=z, p>N_{\varepsilon}+N$ and $F\left(z, x_{j}\right) \in U, a_{j} \leq i \leq b_{j}, j=1,2, \cdots, k$. 
Thus, $F^{0}\left(z, x_{1}\right) \in U$, i.e. $\left(z, x_{1}\right) \in U$. Since $U$ is symmetric, $z \in V$. Thus, every open set $V$ of $X$ contains a periodic point. Hence, the set of periodic points of $T$ is dense in $X$.

Lemma 3.8 ([13]). For a dynamical system $(X, T)$, the following statements are equivalent:

(1) $T$ has the $\bar{d}$-shadowing property (resp., $\underline{d}$-shadowing property);

(2) $T^{k}$ has the $\bar{d}$-shadowing property (resp., $\underline{d}$-shadowing property) for any $k \in N$;

(3) $T^{k}$ has the $\bar{d}$-shadowing property (resp., $\underline{d}$-shadowing property) for some $k \in N$.

Proof of Theorem 3.6. It is well known that every periodic points are minimal points.

By Lemma $3.2(X, T)$ has a dense of minimal points. First, it is presented a proof for the case of the $\bar{d}$-shadowing property. Given any pair of nonempty open subsets $U, V \subset X$, there exist $u \in U \cap M(T)$,

$v \in V \cap M(T)$ and $\varepsilon>0$ such that $B(u, 2 \mathcal{\varepsilon}) \subset U$ and $B(v, 2 \varepsilon) \subset V$. Points $u, v$ are minimal, hence there exists $K>0$ such that for any $n \in N$, so

$[n, n+K] \cap N_{T}(u, B(u, \varepsilon)) \neq \varnothing \neq[n, n+K] \cap N_{T}(v, B(v, \varepsilon))$. There exists $\delta>0$ such that for any $y, z \in X$.

$$
d(y, z)<\delta \Rightarrow d\left(T^{n}(y), T^{n}(z)\right)<\varepsilon \text { for all } 0 \leq n \leq K .
$$

Put $L_{1}=2$ and $L_{n}=L_{n-1}+n$ for $n \geq 2$ and

$$
\mathrm{A}=\mathbb{N} \cap \bigcup_{n \in N}\left[L_{2 n}, L_{2 n+1}\right] \bigcup\left[0, L_{1}\right)
$$

and then denote $\mathrm{B}=\mathbb{N} \bigcup_{n \in N}\left[L_{2 n-1}, L_{2 n}\right]$. It is not difficult to check that $d\left(\mathbb{Z} \backslash\left\{L_{1}, L_{2}, \cdots\right\}\right)=1$, and $d(\mathrm{~A})=d(\mathrm{~B})=\frac{1}{2}$.

Choose a sequence $\left\{x_{i}\right\}_{i=0}^{\infty}$ with

$$
x_{i}= \begin{cases}T^{i}(u), & i \in \mathrm{A}, \\ T^{i}(v), & i \in \mathrm{B} .\end{cases}
$$

Directly by definition, it is obtained that $\left\{x_{i}\right\}_{i=0}^{\infty}$ is a $\gamma$ ergodic pseudo-orbit for any $\gamma>0$ and so by the $\bar{d}-$ shadowing property of $T$, there exists $x \in X$ such that $\bar{d}\left(\Lambda\left(x,\left\{x_{i}\right\}_{i=0}^{\infty}, \delta\right)\right)>\frac{1}{2}$.

But, both sets $\mathrm{A} \cap \Lambda\left(x,\left\{x_{i}\right\}_{i=0}^{\infty}, \delta\right)$ and $B \cap \Lambda\left(x,\left\{x_{i}\right\}_{i=0}^{\infty}, \delta\right)$ are infinite. Therefore, there exists

$$
s \in \mathrm{A} \cap \Lambda\left(x,\left\{x_{i}\right\}_{i=0}^{\infty}, \delta\right) \text { and } t \in \mathrm{A} \cap \Lambda\left(x,\left\{x_{i}\right\}_{i=0}^{\infty}, \delta\right)
$$

such that $K<t-s$.

Clearly, $\quad d\left(T^{s}(x), T^{s}(u)\right)=d\left(T^{s}(x), x_{s}\right)<\delta \quad$ and $d\left(T^{t}(x), T^{t}(u)\right)=d\left(T^{t}(x), x_{t}\right)<\delta$, and points $u, v$ are minimal hence there exist $0 \leq s^{\prime}, t^{\prime} \leq K$ such that $T^{s+s^{\prime}}(u) \in B(u, \mathcal{E})$ and $T^{t+t^{\prime}}(v) \in B(v, \mathcal{E})$. Then it has

$$
d\left(T^{s+s^{\prime}}(u), T^{s+s^{\prime}}(x)\right)<\varepsilon
$$

and

$$
d\left(T^{t+t^{\prime}}(v), T^{t+t^{\prime}}(x)\right)<\mathcal{\varepsilon} .
$$

In particular, $\left(t+t^{\prime}\right)-\left(s+s^{\prime}\right)>0$ and

$$
T^{s+s^{\prime}}(x) \in B(u, 2 \varepsilon) \subset V .
$$

This proves that $(X, T)$ is transitive, hence syndetically transitive by Lemma 3.7.

By Lemma 3.8 , the dynamaical system $\left(X, T^{n}\right)$ has $\underline{d}-$ shadowing for every $n=1,2, \cdots$, which completes the proof of the case of the $\bar{d}$-shadowing property. If $(X, T)$ has the $\underline{d}-$ shadowing property then the same proof works, with the only modification of the definitions

$$
l_{1}=L_{1}=2, l_{n}=2^{l_{1}+\cdots+l_{n-1}}
$$

and

$$
L_{n}=l_{1}+\cdots+l_{n} \text { for } n \geq 2 .
$$

\section{Conclusion}

In the research of dynamical system, because the exact solutions of most systems cannot be obtained, scholars often use numerical calculation. The existence of computational errors inevitably leads to the production of "pseudo-orbit". It is well known that if a system has a "pseudo-orbit-shadowing property in the usual sense", then any pseudo-orbit with a sufficiently small single step error must be tracked by a real orbit-shadowing, and its "shadowing error"is uniformly bounded. This indicates that the numerically calculated pseudo-orbit can truly reflect the local dynamic behavior of the system in a certain sense.

In this paper, it is introduced several types of strong sensitivity and strong transitivity, and discussed the relationship between these properties in systems with different shadowing properties. It will lay a theoretical foundation for further exploring the dynamical behavior of the system.

\section{Acknowledgements}

This work is supported by the Independent Research Foundation of The Central Universities No. DC 201502050201.

\section{References}

[1] Bowen R. (1971). Periodic points and measures for axiom a diffeomorphisms, trans, Trans. Amer. Math. Soc. 154, 377-397. 
[2] Lampart M., Oprocha P. (2009). Shift spaces, $\omega$-chaos and specification property, Topology \& Its Applications 156 (18), 2979-2985.

[3] Kulczycki M., Kwietniak D., Oprocha P. (2013). On almost specification and average shadowing properties, Fundamenta Mathematicae 224 (3), 241-278.

[4] Lidong. W., Hui. W., Guifeng. H. (2014). Minimal sets and $\omega$-chaos in expansive systems with weak specification property, Discrete \& Continuous Dynamical Systems 35 (3), 1231-1238.

[5] Dong Y. (2015). Systems with almost specification property may have zero entropy, Fuzzy Sets \& Systems 364 (10), 53955414.

[6] Kwietniak D., Lacka M., Oprocha P. (2015). A panorama of specification-like properties and their consequences, Contemporary Mathematics arXiv: $1503.07355 \mathrm{v} 2$ [math. DS] 26 May. doi:10.1016/S0031-8. 914 (53) 80099-6.

[7] Kwietniak D., Oprocha P., Rams M. (2016). On entropy of dynamical systems with almost specification, Israel Journal of Mathematics 213 (1), 475-503.
[8] Shah S., Das R., Das T. (2016). Specification property for topological spaces, Journal of Dynamical \& Control Systems $29(7), 1-8$.

[9] Pfister C., Sullivan W. (2007). On the topological entropy of saturated sets, Ergodic Theory and Dynamical Systems 27 (3), 919-956.

[10] Akin E., Glasner E. (2001). Residual properties and almost equicontinuity, Journald' Analyse Mathmatique 84 (1), 243286.

[11] Wen. H., H. Li, Xiangdong. Y. (2012). Family independence for topological and measurable dynamics, Transactions of the American Mathematical Society 364 (10), 5209-5242.

[12] Furstenberg H. (1981). Recurrence in ergodic theory and combinatorial number theory, Princeton University Press.

[13] Xinxing W., Oprocha P., Guanrong C. (2016). On various definitions of shadowing with average error in tracin, Eprint Arxiv 29 (7), 942-1972. 